\section{DEECONOMIAE AGRONEGÓCIO}

ISSN impresso: 1679-1614

ISSN online: $2526-5539$

Vol. 17 | N. 2 | 2019

\section{Carlos Eduardo V. Neves ${ }^{1^{*}}$}

ORCID: $0000-0003-1854-3153$

Geovana Lorena Bertussi'

ORCID: $0000-0001-5929-2536$

1 Universidade de Brasília, Brasília, Distrito Federal, Brasil.

* carlos.vneves@gmail.com

\section{EFICIÊNCIA EM CONCESSÕES DE RODOVIAS FEDERAIS BRASILEIRAS}

\section{RESUMO}

Passados 20 anos desde a primeira etapa de concessões rodoviárias e dado o atual cenário de retomada de crescimento com restrição fiscal, o setor privado deverá ser predominante nos investimentos realizados no segmento rodoviário daqui para frente. Tais investimentos notadamente em infraestrutura - serão fundamentais para esse caminho. Nesse contexto, este trabalho mensurou a eficiência de contratos de concessão de rodovias federais. Foram utilizados dados do período entre 2012 e 2016, referentes à primeira, segunda e terceira etapas de concessões rodoviárias brasileiras, cuja eficiência é calculada e avaliada por meio das técnicas Data Envelopment Analysis (DEA) e Stochastic Frontier Analysis (SFA). A conclusão, por meio dos escores de eficiência, é de que, em média, as concessionárias conseguem produzir $84 \%$ do output máximo possível, sendo que a concessionária mais eficiente entrega $92 \%$ deste. Esse resultado amplia a análise feita por artigos semelhantes, pois considera todos os contratos vigentes nas três etapas de concessões rodoviárias federais. Além disso, fornece insumos para discussão de políticas públicas e para o comportamento do agente regulador (ANTT), que pode atuar incentivando uma maior produtividade das concessionárias.

Palavras-chave: Regulação; Concessões Rodoviárias Federais; DEA; SFA.

\begin{abstract}
After 20 years since the end of the first stage of road concessions and given the current scenario of resumption of growth with fiscal restraint, the private sector should be predominant in the investments made in the road sector from now on. Investments - especially in infrastructure - will be essential for this path. In this context, this study measured the efficiency of current Brazilian federal road concessions. Data from the period between 2012 and 2016 of the contracts for the 1st, 2nd and 3rd stages of Brazilian road concessions were used, whose efficiency is calculated and evaluated through DEA and SFA techniques. The conclusion, through the efficiency scores, is that, on average, the concessionaires are able to produce $84 \%$ of the maximum allowed output, with the most efficient concessionaire delivering $92 \%$. This result extends the analysis made by previous articles, considering all the contracts in the three stages of federal road concessions. In addition, it provides inputs for discussion of public policies and for the behavior of the regulatory authority (ANTT), which can act to encourage a greater productivity of concessionaires.
\end{abstract}

Keywords: Regulation; Federal Road Concessions; DEA; SFA.

JEL Code: C14; O18; R42. 


\section{INTRODUÇÃO}

Em meados dos anos 90, assim como ocorreu em outros setores da economia brasileira, vários trechos de rodovias federais foram objetos de contratos de concessão celebrados com a iniciativa privada. A primeira etapa de concessões terminou em 1998, a segunda etapa ocorreu entre 2008 e 2009, e a terceira etapa se iniciou em 2013, ainda com outros estudos em andamento.

Passados, portanto, 20 anos desde o final da primeira etapa e dado o atual cenário brasileiro de necessidade de retomada do crescimento econômico, que deverá ocorrer mesmo com restrição fiscal por parte do Estado, os recursos do setor privado tenderão a ser predominantes no montante de investimentos realizados no segmento rodoviário nos anos vindouros. Os investimentos - notadamente em infraestrutura - serão de fundamental importância para esse caminho.

Nesse contexto, o presente trabalho mensurou a eficiência das concessões para exploração da infraestrutura rodoviária federal. Foram utilizados dados do período entre 2012 e 2016, compreendendo os contratos referentes à primeira, segunda e terceira etapas de concessões rodoviárias federais, cuja eficiência é calculada e avaliada por meio das técnicas de Data Envelopment Analysis (DEA) e Stochastic Frontier Analysis (SFA).

Além desta introdução, recorre-se ao referencial teórico e à revisão da literatura especializada nas duas seções seguintes, respectivamente. $\mathrm{Na}$ quarta seção, apresenta-se a metodologia a ser utilizada, e prossegue-se à sua aplicação para o caso concreto das rodovias federais concedidas. Em seguida, são apresentados os resultados a partir dos dados então disponíveis. Encerra-se este trabalho com a seção de conclusões.

\section{REFERENCIAL TEÓRICO}

A tarifa consiste em uma contraprestação que os usuários praticam em face da utilização da infraestrutura pública disponibilizada pelo concessionário (GUIMARÃES, 2017). Assim, trata-se de preço arcado pelos usuários, na esfera da relação jurídica contratual que trava com o concessionário, mas é também regulado e controlado pelo poder concedente, em vista de sua ligação estreita com os valores intrínsecos ao serviço público. Além disso, também é elemento que integra a equação econômico-financeira do contrato de concessão, a qual pertence à esfera da relação entre concessionário e poder concedente.

De acordo com a Teoria da Regulação Econômica do Interesse Público (POSNER, 2004), uma vez que o mercado funciona de forma ineficiente, a intervenção do Estado se faz desejável e até necessária. Ao se verificar a existência de uma falha de mercado - um monopólio natural, no caso do serviço de exploração da infraestrutura rodoviária -, a regulação de preços visa a garantir que os usuários não sejam explorados indevidamente pelo concessionário e que a equidade no acesso ao serviço seja assegurada. 
Considerando, então, que os monopólios não regulados tendem a produzir quantidades menores do serviço e cobram preços maiores que aquele que maximizaria o bem-estar, o governo deve intervir de modo a simular um ambiente competitivo que, inclusive, incentive a realização de investimentos por parte do monopolista (PICOT, 2015).

Tirole e Laffont (1993) afirmam que o regulador deve se apoiar exclusivamente nas informações contratuais detidas pelas firmas. As limitações informacionais, contudo, comprometem a eficiência da regulação. Trata-se da chamada assimetria de informações entre regulador e regulado.

Logo, os contratos de concessão devem incluir fortes incentivos, como o mecanismo do preço-teto (price cap), que não são indexados aos custos de produção das firmas, de modo a vencer os problemas de assimetria de informação com os quais o regulador invariavelmente se depara (TIROLE, 2017). Dessa forma, o regulador autoriza uma tarifa máxima e a firma pode escolher seus preços, contanto que estejam abaixo do limite e cubram a totalidade dos seus custos.

O modelo de preço-teto como forma de regulação com alto grau de incentivo pode envolver métodos de reajustamento limitado da tarifa a partir da conjugação de índices de produtividade (GUIMARÃES, Op. Cit.). Uma fórmula prestigiada na experiência britânica pela modalidade price cap é a RPI-X (Retail Price Index menos um fator de produtividade X) ou IPC-X (índices gerais de preços menos um fator de produtividade $X$ ). Ou seja, aplica-se à tarifação um reajustamento segundo um índice geral de preços, limitado à evolução do valor-resultado por um fator de produtividade, que lhe subtrai um percentual arbitrado/calculado pelo poder concedente regulador.

Agrell e Bogetoft (2013) afirmam que uma das áreas mais proeminentes para aplicação das técnicas de benchmarking é justamente a que se refere à regulação de monopólios naturais, uma vez que tais técnicas podem informar se determinada regulação produz efeitos econômicos em usuários e firmas reguladas de modo equilibrado.

O benchmarking - comparação do desempenho relativo entre empresas - é uma técnica bastante utilizada por reguladores de diversos países, como Noruega, Áustria, Finlândia, Holanda e Alemanha, na regulação dos mais variados tipos de serviços associados à infraestrutura. O objetivo da técnica é extrair uma métrica de desempenho relativo entre as empresas do setor regulado, de modo que possam ser identificadas aquelas mais eficientes. A eficiência relativa é então convertida em Fator $X$, o qual será aplicado na equação tarifária de cada empresa, de maneira que aquelas menos eficientes tenham um Fator $X$ maior, o que resulta na diminuição do índice de reajuste tarifário ao qual faria jus naquele período.

A ideia é que, ao final do próximo ciclo regulatório, aquelas empresas apontadas como menos eficientes sejam incentivadas a melhorar o seu desempenho, de modo que, na próxima aplicação do Fator $X$, possam figurar entre as mais eficientes, para então conseguirem um maior índice de 
reajustamento tarifário. Portanto, o mecanismo descrito tende a equilibrar - de um lado - o ímpeto de maximização da receita pelas empresas reguladas (minimização do Fator $X$ ) e - de outro - a produção de melhores resultados para os usuários daquele serviço público.

O que é apresentado neste trabalho é exatamente um modo objetivo de cálculo da eficiência de diferentes concessionárias de rodovias na prestação do serviço de infraestrutura. Com esse parâmetro de eficiência, por sua vez, o regulador poderá compartilhar parte dos ganhos de produtividade do concessionário com os usuários do referido serviço, promovendo incentivos tanto do ponto de vista de melhores práticas e absorção tecnológica por parte das concessionárias, quanto pela perspectiva de uma maior universalização e qualidade do serviço prestado aos usuários das rodovias federais brasileiras.

\section{REVISÃO DA LITERATURA EMPÍRICA}

Farsi e Filippini (2004) estimaram, por meio de diferentes métodos paramétricos, a eficiência de custos de empresas de distribuição de energia na Suíça. O modelo de fronteira foi calculado utilizando quatro métodos: Displaced Ordinary Least Squares (DOLS), Fixed Effects (FE), Random Effects (RE) e Maximum Likelihood Estimation (MLE). O banco de dados retratou um painel desbalanceado no período de 1988 a 1996 em 59 concessionárias. Os resultados apontam para um melhor encaixe do modelo de FE na estimação das características da função de custo. Além disso, existe uma consistência mútua entre os resultados de eficiência obtidos a partir dos diferentes métodos. As diferenças entre os métodos aparecem, em particular, nas companhias que são tidas como as mais e as menos eficientes. Por isso, os autores recomendam que o benchmarking deve ser utilizado considerando diferentes modelos, com o objetivo de ser possível avaliar a consistência dos resultados.

A avaliação de desempenho de concessionárias ou serviços em infraestrutura de transportes pode utilizar técnicas para estimar funções de custo como sendo o fator de comparação entre elas, conforme apontado por Massiani e Ragazzi (2008) e Smith (2012). Outros autores preferem empregar funções de produção (ISMAEL DE AZEVEDO et al., 2012). Também é possível utilizar uma relação mais completa entre insumos e produtos, considerando ainda alguns aspectos relativos à qualidade dos serviços e à infraestrutura disponível (DIANA, 2010). Outros trabalhos, por sua vez, avaliam a eficiência sob um enfoque específico, como o da eficiência técnica, uma relação entre a infraestrutura disponível e a sua utilização (SCOTTI et al., 2012).

Wang e Tsai (2009) afiançam que toda organização lucrativa irá estabelecer o máximo ganho como seu objetivo primário e lutará para obter uma eficiência produtiva otimizada na conversão de "entradas" (inputs) em "saídas" (outputs). Então, segundo os autores, os indicadores financeiros podem prover uma base para avaliação da eficiência produtiva. Contudo, empresas estatais responsáveis por realização de serviços de manutenção 
em rodovias, servindo como organizações sem fins lucrativos, não podem ser avaliadas exclusivamente por meio de indicadores financeiros.

Assim, Wang e Tsai estudaram o desempenho de 31 trechos de manutenção de rodovias pertencentes ao Directorate General of Highways (DGH). As variáveis de entrada consideradas foram o orçamento total, a força de trabalho e as máquinas utilizadas, e as de saída foram a extensão da rodovia, o gasto ordinário em manutenção, os gastos emergenciais e a receita proveniente de utilização da faixa de domínio. Os pesquisadores então utilizaram o DEA-CCR e DEA-BCC para estimar a eficiência técnica, a produtiva e a de escala, que serviram para propor melhorias nos serviços de manutenção de rodovias.

Possamai (2006) utilizou o DEA para avaliar a eficiência técnica de concessionárias de rodovias do Rio Grande do Sul. As análises tiveram como foco a evolução das concessionárias em um período de quatro anos. Em relação ao DEA, os modelos foram orientados para a redução na utilização de insumos, considerando tecnologia de retornos variáveis e questões relativas à escala. As análises realizadas pelo autor tão somente permitiram identificar que não há homogeneidade na gestão das empresas nos diferentes polos, uma vez que os resultados foram distintos em termos de eficiência empresarial, de imagem e de segurança. Assim, há baixa correlação entre ser eficiente do ponto de vista de servir ao usuário e de ser eficiente na obtenção do lucro.

Clímaco et al. (2010) estudaram, por meio do DEA, o desempenho de algumas rodovias concedidas no Brasil, estendendo os resultados de Gomes et al. (2004). Como o número de rodovias analisado era pequeno, os autores recorreram ao modelo MCDEA de Li e Reeves (citados por Clímaco et al., 2010), adequado para resolver esse tipo de problema. Ainda, sugeriram a extensão do modelo CCR considerando duas funções objetivo extras. Os resultados, de acordo com os autores, permitiram verificar a percepção dos usuários acerca do alto valor do pedágio da Ponte Rio-Niterói em relação ao seu desempenho relativo.

Welde e Odeck (2011) analisaram o nível de eficiência das companhias que administram as rodovias pedagiadas da Noruega no período de 2003 a 2008. Para isso, utilizaram o DEA e o SFA. O estudo concluiu que existe grande variação de eficiência entre as empresas, e que as maiores tendem a ter melhor desempenho. Os autores também verificaram que existem evidências de ganhos de escala não explorados, e que as empresas melhoraram seu desempenho ao longo do tempo devido à evolução das tecnologias de cobrança de pedágio.

Por sua vez, Gomes et al. (2012), ao analisarem as concessões federais, usaram o modelo DEA-CCR sob o argumento de não haver evidências de ganhos de escala no setor. Os autores avaliaram cinco rodovias federais pedagiadas e usaram dois modelos parciais e dois modelos globais - que agregam os resultados dos modelos parciais -, com variáveis relativas à arrecadação das praças de pedágio, ao tráfego, ao número de acidentes, aos investimentos realizados e à extensão total da rodovia. Os resultados obtidos indicaram que essa abordagem pode ser empregada com sucesso 
na determinação de um índice único de qualidade, além de permitir verificar uma tendência de melhoria das eficiências das concessionárias ao longo do tempo.

Correa (2012) utilizou o DEA com o objetivo de comparar a eficiência no transporte ferroviário e rodoviário na Colômbia. As Decision Making Units (DMUs) escolhidas foram os tipos de caminhões e vagões. A avaliação foi feita tanto com o modelo CRS como com o VRS, sendo que o segundo apresenta muitas DMUs eficientes $(100 \%)$, o que dificultou a comparação. Além dessa análise, considerou também apenas os caminhões e os vagões. A conclusão do artigo é que o transporte ferroviário, em média, é mais eficiente que o rodoviário, apresentando um índice de 74,4\% contra 20,6\%. Assim sendo, a sugestão é de que seja feito investimento na infraestrutura ferroviária do país para o transporte de mercadorias.

O modelo DEA-BCC é utilizado por Ismael de Azevedo et al. (2012), a fim de avaliar a eficiência temporal de rodovias federais brasileiras concedidas entre os anos de 2005 e 2008. São avaliadas seis concessionárias, sendo que cada uma, em cada ano, foi considerada como uma DMU distinta. Dois modelos DEA foram empregados: o primeiro visou a medir a eficiência da concessionária que administra a rodovia com relação ao uso de bens de capital e à gestão de pessoal na operacionalização dos serviços; enquanto o segundo avaliou a eficiência da rodovia com relação ao uso de receitas e investimentos em segurança. Os resultados mostraram que a Nova Dutra, em ambos os modelos, possui índice de eficiência sempre crescente, atingindo a máxima eficiência no ano de 2008. Quatro DMUs foram globalmente eficientes: Ecosul em 2005, Nova Dutra em 2008 e Ponte S.A. em 2007 e 2008.

Ferreira et al. (2013) aplicaram o modelo DEA-BCC com orientação a outputs, para análise da eficiência de rodovias concedidas sob a perspectiva do usuário da rede de transporte. Como resultado, seis rodovias foram eficientes e oito ineficientes.

Medeiros (2014) apresentou uma discussão sobre quais modelos regulatórios permitem o uso do benchmarking como ferramenta auxiliar na decisão e uma proposta de uso para o cálculo do fator " $X$ ". Realizou um estudo de caso sobre as concessionárias brasileiras de rodovias federais da segunda etapa, visando a obter o fator de eficiência de tais empresas, amparado nos métodos de análise estocástica de fronteira (SFA) e de análise envoltória de dados (DEA). Segundo o autor, a utilização de poucas DMUs foi um dos motivos para que o método apresentasse resultados inconclusivos para o caso estudado.

Profeta (2014) estudou os vários modelos de concessões de rodovias adotados no Brasil, pretendendo comparar a eficiência de cada um. Para tanto, adotou como metodologia a análise envoltória de dados (DEA-BCC) em dois estágios com uso de regressão tobit bootstrap. Os resultados revelaram que os modelos federal, gaúcho e paulista foram os que se apresentaram mais eficientes em relação aos demais. Além disso, segundo a autora, a participação das agências reguladoras configura fator importante para explicar os níveis de eficiência do setor. 
Salgado et al. (2016) avaliaram a eficiência na prestação do serviço de 14 concessionárias de rodovias federais, com foco na diferença entre as eficiências da primeira e segunda etapas do programa de concessão federal. Os autores utilizaram a análise envoltória de dados orientada a produto (output), com dados do período de 2010-2013, contendo informações de 14 concessões de rodovias, cinco pertencentes à primeira etapa e oito pertencentes à segunda etapa. Como insumos, foram utilizados o custo operacional por quilômetro e o fluxo de veículos; e como produto, foram utilizados o inverso do número de acidentes por quilômetro, o inverso da tarifa cobrada como pedágio, o número de atendimentos por quilômetro e o inverso da quantidade de Termos de Registro de Ocorrências (TROs). Os resultados mostram que as concessões da segunda etapa apresentaram um melhor nível de eficiência na prestação dos serviços em comparação com a primeira.

O presente trabalho, portanto, avança em relação à literatura, pois avalia também as três etapas de concessões no período entre 2012 e 2016, além de aplicar várias técnicas para a verificação de consistência dos resultados: a análise envoltória de dados orientada a output, considerando retornos variáveis de escala (DEA-VRS); a análise DEA de $2^{\circ}$ Estágio, levando em consideração as variáveis ambientais; e a aplicação de vários modelos SFA.

\section{METODOLOGIA}

\section{Dados do Modelo}

Um dos pontos mais sensíveis na realização da análise de benchmarking em concessões é a escolha das variáveis de input e output do modelo. É recomendável que se utilize prioritariamente variáveis que reflitam o uso de recursos na atividade sob análise (JAMASB; POLLITT, 2000).

O tratamento de dados não é estatístico, comparando o desempenho de uma empresa específica com a atuação real de outras firmas. No entanto, o modelo é sensível à escolha de variáveis de insumos e produtos. Também responde à quantidade de insumos e produtos utilizados na análise: quanto maior a quantidade agregada de insumos e produtos frente às DMUs, maior o número destas que serão consideradas eficientes.

Os critérios aqui utilizados para a escolha das variáveis foram:

- $\quad$ facilidade de obtenção;

- $\quad$ facilidade de interpretação no contexto;

- $\quad$ transparência, uma vez que a informação deve ser preferencialmente pública;

- $\quad$ confiabilidade (preferencialmente deve já ter sido auditada).

Assim, como um primeiro modelo de avaliação, foram selecionadas as variáveis definidas a seguir, em conformidade com o que foi traçado por Profeta (Op. Cit.). 
Como outputs: avaliação geral do trecho concedido (AVG), de acordo com as pesquisas anuais da Confederação Nacional dos Transportes. Este estudo tem como objetivo avaliar as condições das rodovias pavimentadas brasileiras segundo a segurança e o desempenho observados pelos usuários, identificando os aspectos das vias que os afetam, direta ou indiretamente - em relação ao Pavimento, à Sinalização e à Geometria da Via. O resultado da análise combinada desses três elementos indica a classificação do Estado Geral das rodovias em péssimo, ruim, regular, bom e ótimo. No presente trabalho, utilizou-se a escala de um a cinco (nos moldes de uma escala Likert) para quantificar a avaliação dada para cada concessão. A nota de cada subtrecho dentro do trecho correspondente à rodovia concedida foi ponderada de acordo com a respectiva extensão para então resultar na nota geral da concessão.

Como inputs: receitas totais (RT), obtidas a partir das demonstrações financeiras auditadas das companhias e disponibilizadas na página oficial da ANTT, divididas pelas respectivas extensões (EXT); e custos totais (CT), obtidos a partir das demonstrações financeiras auditadas das companhias e disponibilizados na página oficial da ANTT, divididos pelas respectivas extensões (EXT).

Note-se que o modelo pode ser considerado bastante simples, pois se trata de um modelo com apenas dois inputs e um output. No caso, sendo utilizada a orientação a output, o que se pretende é medir a eficiência das concessionárias quanto à gestão de suas receitas e custos em relação ao resultado percebido em termos de qualidade da rodovia. Assim, será mais eficiente a concessionária que apresentar o melhor desempenho operacional.

Ainda sobre os dados, vale dizer que a ANTT não dispõe de um banco de dados organizado, de onde se possa simplesmente retirar as informações necessárias para a simulação de qualquer modelo. Normalmente, os dados sobre as concessionárias estão dispersos em várias planilhas, manipuladas apenas pelos especialistas que os utilizam para a realização das revisões tarifárias e fiscalizações econômico-financeiras. Espera-se que, com a necessidade de realizar a análise de benchmarking, haja o impulso necessário para a melhor organização dos dados sobre as concessionárias de rodovias.

Outro aspecto importante sobre os dados é que existem três etapas de concessões e, ao todo, vinte e um contratos (vinte ativos e um encerrado). Portanto, o número de observações pode ser considerado restrito, em uma primeira avaliação. Por outro lado, é importante considerar a peculiaridade de, ao contrário da grande maioria dos estudos, estar realizando-se a análise não de uma amostra, da qual se pretende inferir resultados. Na verdade, avalia-se a própria população (finita, pois a amostra possui o mesmo tamanho da população) naquele determinado período. Em termos práticos, tem-se, por exemplo, que a média amostral é igual à média populacional e a variância da média é nula. Assim, no caso de uma regressão por meio de mínimos quadrados ordinários (MQO), por exemplo, o estimador de MQO permanecerá válido (BUSSAB; MORETTIN, 2017 e NETO, 2017). 
É possível trabalhar com dados em painel na análise de eficiência, assumindo que o resultado de cada concessionária, em cada ano, representa uma DMU distinta, o que aumenta substancialmente o tamanho da amostra. Porém, nesse caso, não se pode deixar de considerar que existe correlação e colinearidade entre os dados dos consecutivos anos, o que demanda também aplicação de outras técnicas mais sofisticadas.

Por outro lado, a escolha das variáveis do modelo com apenas um output e dois inputs está adequada ao pequeno número de dados. Embora não haja consenso na literatura especializada sobre o número mínimo de observações, aconselha-se que este seja o maior possível, ou que, pelo menos seja o quíntuplo do número de variáveis (TIRYAKI; ANDRADE, 2017). Gomes e Batista (2004) alegam que o número de variáveis não deve ser superior à terça parte do número de observações. Por fim, a chamada "Regra de Ouro" (BANKER et al., 1989) recomenda que, para utilizar os modelos DEA (CCR e BCC), o número de DMUs deve ser pelo menos igual a três vezes a soma total do número de variáveis (inputs e outputs) envolvidas, ou pelo menos igual ao produto do número de variáveis de input e output, adotando-se o critério associado ao maior número de DMUs necessárias.

Ante o exposto, mesmo sabendo que um conjunto pequeno de observações não é o ideal, entende-se que os resultados das simulações podem ser considerados válidos. Para diminuir eventuais distorções nas observações, que consideram conjuntamente os dados das três etapas de concessões, os inputs e os outputs são calculados como a média dos últimos cinco anos. Mais adiante, será demonstrado que tal aproximação é adequada ao conjunto dos dados.

Além do custo total médio por quilômetro (custm), da receita total média por quilômetro (recm) e da avaliação geral do trecho (avgm), foram utilizadas outras variáveis para verificar em que medida fatores externos poderiam interferir na eficiência calculada. Nesse caso, são consideradas como variáveis exógenas: o número de praças de pedágio por concessão (npp), a extensão do trecho concedido (ext), a tarifa média a cada $100 \mathrm{~km}$ (tarm) e duas dummies, dEtapa2 e dEtapa3, que assumem o valor 1 quando a DMU pertence, respectivamente, à segunda e à terceira etapa, e 0 no caso contrário.

\section{Modelos DEA e SFA}

A ideia do modelo pioneiro de Charnes-Cooper-Rhodes (CCR ou CRS) é escolher para cada DMU o conjunto de preços de insumos e produtos para os quais é obtida a máxima razão entre os produtos e os insumos ponderados, sujeitos a algumas restrições:

- Todos os preços de insumos e produtos devem ser não negativos;

- $\quad$ Para todas as DMUs, a soma ponderada dos produtos não deve exceder a soma ponderada dos insumos. 
O problema pode, então, ser apresentado como um problema de programação linear. A imposição da restrição de convexidade é o que diferencia os modelos de retornos constantes (CRS) e de retornos variáveis (VRS). Assim, a eficiência de escala pode ser mensurada a partir dos índices calculados considerando os modelos CRS e VRS. Caso haja alguma diferença de eficiência técnica sob CRS e VRS, então existirá um indicativo de ineficiência de escala.

Os escores de eficiência calculados podem ainda ser avaliados de modo que se busque entender quais fatores exógenos (ambientais) afetam a eficiência de cada DMU. Nesse chamado "segundo estágio" da análise DEA, os escores são regredidos contra as variáveis exógenas que se entende relevantes.

A escolha de um modelo para realizar a regressão de segundo estágio no DEA não é tarefa trivial. A técnica econométrica mais utilizada é a regressão tobit (limite superior igual a 1 e limite inferior igual a 0), mas também podem ser utilizados métodos mais sofisticados, como o proposto por Simar e Wilson (2007).

Neste trabalho, será adotado o método de Simar e Wilson (Op. Cit.), no qual os autores assumem que a consistência dos parâmetros da regressão no segundo estágio não é afetada pelo uso de estimativa para os escores de eficiência no lugar dos seus reais valores. Duas hipóteses elaboradas por Simar e Wilson são particularmente relevantes (RAMALHO; RAMALHO; HENRIQUES, 2010). Primeiro, eles entendem que as variáveis exógenas não afetam a fronteira de eficiência. Segundo, assumem que os reais escores de eficiência seguem uma distribuição normal truncada. O resultado final do algoritmo será, então, o vetor de escores de eficiência corrigidos de acordo com as variáveis exógenas.

Seguindo o princípio da parcimônia (COELLI et al., 2005), segundo o qual deve ser escolhida a forma funcional mais simples e que, ao mesmo tempo, consiga produzir bons resultados, neste trabalho será utilizada a função Cobb-Douglas. Após a escolha da função de produção, a estimativa do modelo de fronteira estocástica passa, primeiramente, pela estimação dos parâmetros da função de fronteira e, posteriormente, pelo cálculo do valor da ineficiência para cada unidade.

Existem várias formas de se estimar a função de fronteira estocástica. Uma abordagem se baseia na não definição a priori de uma função de distribuição para os componentes de erro (distribution-free approach - DFA). Outra abordagem assume hipóteses específicas sobre a função de distribuição dos erros e aplica o método da máxima verossimilhança (parametric approach - PA). Ambas serão utilizadas aqui.

\section{Dos escores de eficiência}

Reguladores noruegueses e holandeses, reconhecendo a limitação prática de se alcançar elevados ganhos de eficiência, mesmo sob incentivos regulatórios, impuseram limites de eficiência a serem demandados, 
principalmente em relação a companhias menos eficientes (JAMASB; POLLITT, 2000).

$\mathrm{Na}$ Alemanha (BOGETOFT; OTTO, 2010), para a regulação das concessionárias de distribuição de energia elétrica, foi adotado um modelo de benchmark orientado a input, baseado na ideia de minimização dos valores de insumos e manutenção do nível de produção. Nesse caso, o regulador alemão optou por simular o nível de eficiência de cada concessionária por meio do máximo entre quatro escores: $\operatorname{EDEA}(\mathrm{B})$, $\operatorname{EDEA}(\mathrm{S}), \operatorname{ESFA}(\mathrm{B})$ e $\operatorname{ESFA}(\mathrm{A})$; em que EDEA é o escore de eficiência de Farrell calculado mediante DEA com retornos de escala não decrescentes, e ESFA é o escore de eficiência calculado por meio do SFA orientado a input. Os argumentos B e S representam os valores de livro texto e os valores padronizados, respectivamente. Às empresas que demonstram um nível particularmente baixo de eficiência é atribuído um nível mínimo de 0,60 (ou $60 \%$ ). Nesse caso, o regulador enviesa a sua análise em prol do regulado, em face da sua avaliação de risco no cálculo dos custos padronizados.

No presente trabalho, propõe-se metodologia semelhante à do órgão regulador alemão. Serão calculados primeiramente os escores de eficiência utilizando o modelo DEA-VRS (simples, bootstrap com correção de viés DEAbc -, e com aplicação do método de Simar-Wilson - DEAsw) e o SFA (modelos Corrected OLS - COLS -, Corrected Mean Absolute Deviation CMAD -, Half-Normal, Exponential). Posteriormente, o escore individual será definido a partir da média aritmética dos escores calculados por meio dos diversos métodos. A exemplo do regulador alemão, adotar-se-á o escore mínimo de 0,50 (ou 50\%). Porém, a média aritmética é calculada somente considerando os valores de eficiência que atendam ao critério para não classificação como outliers. Em resumo, o conjunto de equações para o cálculo do escore de eficiência de cada firma é o seguinte:

$$
\begin{aligned}
& E_{i}=\max \left\{\bar{E}^{i} ; 0,50\right\}, \\
& E_{i} \in[0,50 ; 1,00] \\
& E_{i}=\frac{1}{n} \sum_{k=1}^{n} E_{k}^{i}, \text { sujeito a } \\
& \rho_{E_{D E A^{i}}{ }^{i} E_{k}}{ }^{i}>|0,5| e \\
& E_{k}{ }^{i} \in\left\{E_{D E A-" \text { puro" }^{i}}{ }^{i} ;{E_{\text {DEAbc }}}^{i} ;{E_{\text {DEAsw }}}^{i} ;{E_{\text {SFAcols }}}^{i} ;{E_{\text {SFAcmad }}}^{i} ;{E_{\text {SFAhn }}}^{i} ; E_{\text {SFAexp }}{ }^{i}\right\}
\end{aligned}
$$

De acordo com a formulação proposta, a média aritmética será composta apenas por aqueles escores de eficiência cuja correlação $\rho_{E_{D E A}{ }^{i}, E_{k}}{ }^{i}$ seja maior que 0,5 em relação ao escore calculado por meio do DEA simples. Esse critério de exclusão de outliers visa a garantir que haja mínima coerência entre os escores calculados de acordo com as diversas metodologias. Vale lembrar que a média aritmética é sempre a maior das três médias pitagóricas (média aritmética, média harmônica e média geométrica). 


\section{RESULTADOS E ANÁLISE}

Para avaliar a eficiência das concessionárias de rodovias federais, optou-se por um modelo orientado a outputs, tendo como variáveis de entrada (inputs) cust_km e rec_km, e como variável de produto (output), avg. Vale frisar que, conforme conclusões da seção anterior, optou-se por adotar a média dessas variáveis no período avaliado. Nesse tipo de análise, o que se está buscando é a maximização do produto, mantendo constantes as entradas.

O sumário da análise DEA, considerando retornos variáveis de escala (DEA-VRS), pode ser visto na Tabela 1. Os três escores distintos representam os resultados da análise DEA "simples" (deascore_o), da análise DEA bootstrap (deascore_bc) e da análise DEA utilizando o procedimento de Simar-Wilson (deascore_sw).

Tabela 1. Sumário dos resultados da análise DEA-VRS

\begin{tabular}{cccccc}
\hline Modelo & Média & Desv. Pad. & p50 & Mín. & Máx. \\
\hline deascore_o & 0,9185 & 0,0784 & 0,9134 & 0,7190 & 1,0000 \\
\hline deascore_bc & 0,9452 & 0,0839 & 1,0000 & 0,6794 & 1,0000 \\
\hline deascore_sw & 0,9156 & 0,0902 & 0,9603 & 0,7127 & 1,0000 \\
\hline
\end{tabular}

Fonte: Elaborado pelos autores.

O cálculo dos escores de eficiência, por meio dessas três formulações do DEA, permite ao regulador ter maior segurança quanto à consistência dos resultados.

O DEA em dois estágios (adotando o procedimento de Simar-Wilson) permite corrigir os escores por meio de variáveis ambientais (Tabela 2), que acabam por impor às concessionárias uma espécie de "ambiente médio". Assim, as diferenças de eficiência tenderão a estar quase que exclusivamente relacionadas à eficiência gerencial de cada empresa.

Tabela 2. Resultado da regressão no segundo estágio do modelo DEA

\begin{tabular}{lcc}
\hline \multicolumn{1}{c}{ Variável } & tob_out1 & sw_out1 \\
\hline scorebc & & \\
\hline $\mathbf{n p p}$ & $0,14599708^{* * *}$ & $0,16011687^{* * *}$ \\
\hline ext & $-0,00142473^{* * *}$ & $-0,00162082^{* * *}$ \\
\hline tar & $0,02600594^{* *}$ & $0,02784542^{* * *}$ \\
\hline dEtapa2 & $-0,12441263^{*}$ & $-0,12042088^{*}$ \\
\hline dEtapa3 & $-0,06218078$ & $-0,07962789$ \\
\hline legenda: & ${ }^{*} \mathrm{p}<.2 ;{ }^{* *} \mathrm{p}<.1 ;^{* * *} \mathrm{p}<.05$ & \\
\hline
\end{tabular}

Fonte: Elaborado pelos autores.

Na coluna "tob_out1", constam os resultados do segundo estágio, baseado no que a literatura chama de "análise ingênua", pois tão somente se faz a regressão truncada dos resultados de eficiência, o que gera diversos 
problemas na estimação dos parâmetros, especialmente em pequenas amostras (SIMAR; WILSON, Op. Cit.).

Por isso, o algoritmo de Simar-Wilson é bastante adequado para a geração de estimativas mais consistentes, como aquelas que constam da coluna "sw_out1". Nesse caso, é possível verificar que somente as variáveis referentes a número de praças de pedágio (npp), extensão (ext) e tarifa (tar) são significativas a 5\%. É interessante notar também que as dummies para os contratos da segunda e terceira etapas de concessão não são significativas estatisticamente a 5 ou $10 \%$.

De acordo com a avaliação em tela, aparentemente a diferença entre etapas não foi significativa para a ineficiência das concessionárias. Os coeficientes calculados indicam que o número de praças de pedágio e a tarifa tem efeito positivo no valor de ineficiência (diminuem a eficiência), enquanto que a extensão tem efeito negativo (aumenta a eficiência). Assim, pode-se dizer que, ceteris paribus, uma unidade a mais de praça de pedágio aumenta a ineficiência em aproximadamente $16 \%$ (diminui a eficiência). A tarifa mais alta em uma unidade também aumenta o escore de ineficiência em 2,78\% (diminui a eficiência). Já a extensão, em que pese diminuir a ineficiência, tem impacto de baixa magnitude.

O resultado da análise em dois estágios, demonstrado na Tabela 3, considera como eficiente aquela DMU que tem o valor mais próximo de 1 (ao contrário da medida tradicional, conforme consta da Tabela 1, cujos resultados de deascore_sw e deascore_bc foram ajustados para o intervalo entre 0 e 1). Na Tabela 3, estão as estatísticas dos resultados do primeiro (score) e segundo (ohat) estágios da análise DEA. Nota-se que as concessões da segunda etapa são as que recebem maior influência das variáveis ambientais, cuja média sofreu um aumento de mais de 7\% nos escores de eficiência, enquanto que nas outras etapas houve uma redução de até 3,18\%. Ademais, as concessões da terceira etapa apresentam desempenho médio muito próximo daquelas da segunda etapa, porém com menor influência das variáveis de ambiente.

Tabela 3. Resultado da análise DEA por etapa de concessão

\begin{tabular}{|c|c|c|c|c|c|c|}
\hline Etapa & Variável & Obs. & Média & Desv. Pad. & Mín. & Máx. \\
\hline \multirow{3}{*}{$\begin{array}{c}\mathbf{1}^{\mathrm{a}} \\
\text { Etapa }\end{array}$} & score & 6 & 1,1178 & 0,1434 & 1,0000 & 1,3907 \\
\hline & ohat & 6 & 1,1496 & 0,1840 & 0,9057 & 1,4029 \\
\hline & Diferença & & $-3,180 \%$ & & & \\
\hline \multirow{3}{*}{$\begin{array}{c}2^{\mathbf{a}} \\
\text { Etapa }\end{array}$} & score & 8 & 1,1072 & 0,0954 & 1,0000 & 1,2473 \\
\hline & ohat & 8 & 1,0319 & 0,1067 & 0,8423 & 1,1694 \\
\hline & Diferença & & $7,530 \%$ & & & \\
\hline \multirow{3}{*}{$\begin{array}{c}3^{\mathrm{a}} \\
\text { Etapa }\end{array}$} & score & 7 & 1,0353 & 0,0673 & 1,0000 & 1,1821 \\
\hline & ohat & 7 & 1,0596 & 0,1757 & 0,7532 & 1,3012 \\
\hline & Diferença & & $-2,430 \%$ & & & \\
\hline
\end{tabular}

Fonte: Elaborado pelos autores. 
Conforme destacado anteriormente, a análise utilizando mais de um método tende a permitir que seja verificada a consistência do modelo proposto. Ao se optar pela análise SFA, é possível avaliar se a forma funcional, considerando os parâmetros de entrada e saída, gera resultados adequados com a teoria de fronteira estocástica.

Coelli (1995) propôs um teste sobre os resíduos de uma regressão por Mínimos Quadrados Ordinários (MQO), com o intuito de checar a validade da especificação do modelo de fronteira estocástica (e, por sua vez, do modelo DEA). Essa análise deve verificar se há assimetria negativa na distribuição dos resíduos para a função de produção da fronteira estocástica.

Aplicando tal teste ao modelo proposto neste trabalho, o resultado obtido é -1,1404417 (o valor crítico é 1,96), ou seja, a avaliação indica que os resíduos são assimétricos para a esquerda (a hipótese nula é rejeitada) e, portanto, o modelo de fronteira estocástica está corretamente especificado. Uma vez que foi encontrado suporte para o modelo de fronteira estocástica especificado, pode-se proceder com a estimativa, considerando válidas as hipóteses sobre a distribuição do erro composto.

Assim, constam da Tabela 4 os resultados da análise de fronteira estocástica utilizando os métodos de Mínimos Quadrados Ordinários (OLS), Mínimos Quadrados Ordinários Corrigidos (COLS), Mínimos Quadrados Ordinários Corrigidos de acordo com a mediana (CMAD), Fronteira Estocástica com a função de distribuição half-normal (sfa_hn), Fronteira Estocástica com a função de distribuição exponencial (sfa_exp), Fronteira Estocástica com a função de distribuição half-normal com variáveis exógenas (sfa_hn_ex) e Fronteira Estocástica com a função de distribuição exponencial com variáveis exógenas (sfa_exp_ex).

Tabela 4. Resultado da análise SFA

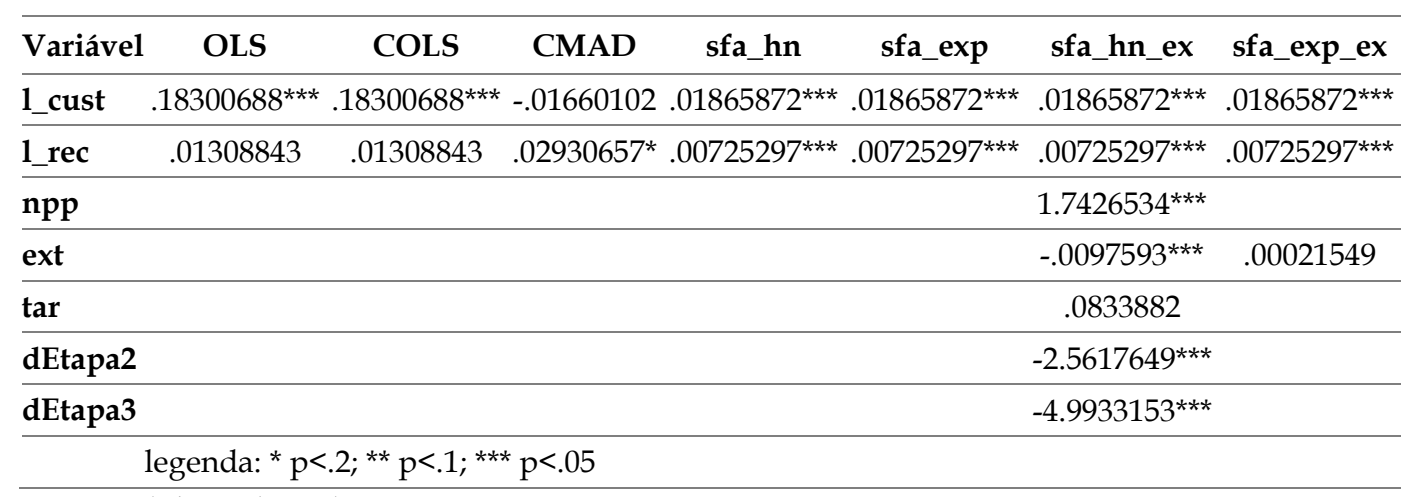

Fonte: Elaborado pelos autores.

Ao se comparar as diversas estimativas, verifica-se que o coeficiente para a variável referente ao custo é aproximadamente o mesmo, exceto para o modelo CMAD. Por outro lado, ao se comparar os diversos modelos de fronteira estocástica, as variáveis de input, além de serem significativas a $5 \%$, são rigorosamente as mesmas, o que remete à consistência das 
estimativas. Além disso, no modelo sfa_hn_ex, todas as variáveis ambientais, exceto tarifa, são relevantes para a determinação do escore de eficiência.

A elasticidade da avaliação média da concessão em relação ao custo é de aproximadamente $1,87 \%$, enquanto que a elasticidade da avaliação média da concessão em relação à receita é de aproximadamente 0,73\%.

As variáveis exógenas, por sua vez, devem afetar o desempenho dos produtores, seja por meio da sua influência na estrutura tecnológica utilizada para converter os insumos em produtos, seja por meio da sua influência na eficiência com a qual os insumos são convertidos em produtos.

De acordo com Kumbhakar et al. (2015), tal abordagem foi inicialmente incentivada a partir dos estudos acerca dos problemas de heterocedasticidade na variável aleatória $u_{i}$. Porém, verificou-se que o trabalho poderia ser aplicado também à análise dos determinantes exógenos da ineficiência. Ainda de acordo com os autores, diferentemente do DEA, no caso do SFA, a prática recomendada, devido aos problemas relacionados ao viés do estimador, é que se utilize a análise em um único estágio (KUMBHAKAR; WANG; HORNCASTLE, Op. Cit.). Tal procedimento foi o aqui adotado.

Quanto às variáveis exógenas, foram significativas a 5\% a extensão (ext), o número de praças de pedágio (npp) e, diferente do modelo DEA, as dummies de etapa (dEtapa2 e dEtapa3).

Ao se analisar o sinal das variáveis exógenas, nota-se que estas se comportam de modo semelhante ao segundo estágio da análise DEA. O número de praças de pedágio e a tarifa (apesar de estatisticamente insignificante) contribuem positivamente para a ineficiência (ou negativamente para a eficiência), enquanto que a extensão contribui negativamente. Ao mesmo tempo, os coeficientes das dummies de etapa mostram que o fato de a concessão pertencer à segunda ou à terceira etapa implica um efeito negativo na ineficiência (ou positivo na eficiência). Esse resultado sugere um aprendizado ao longo das fases de concessões rodoviárias federais brasileiras, com avanços na eficiência medida nas etapas mais recentes em relação à primeira, realizada ainda na segunda metade dos anos 90. Ademais, esse desdobramento está em consonância com aquele verificado por Salgado et al. (2016), que também encontraram evidências de melhor eficiência na segunda etapa em relação à primeira.

A magnitude dos efeitos marginais das variáveis explanatórias da ineficiência, por sua vez, não pode ser avaliada diretamente a partir dos resultados, dado que existe uma relação não linear entre os coeficientes calculados e as variáveis. Como neste trabalho interessa muito mais verificar a coerência dos cálculos entre os diversos modelos DEA e SFA, não será avaliada a magnitude desses coeficientes.

Estimadas as equações para a fronteira estocástica, são então calculados os resíduos e, a partir destes, é obtido o escore de eficiência. Os resultados dessa operação estão na Tabela 5. 
Tabela 5. Escores de Eficiência de acordo com a análise SFA

\begin{tabular}{cccccc}
\hline Variável & Obs. & Média & Desv. Pad. & Mín. & Máx. \\
\hline eff_ols & 21 & 1,03610 & 0,19123 & 0,69127 & 1,52612 \\
\hline eff_cols & 21 & 0,67891 & 0,12530 & 0,45296 & 1,00000 \\
\hline eff_cmad & 21 & 0,88238 & 0,07991 & 0,69883 & 1,00000 \\
\hline eff_hn & 21 & 0,78669 & 0,07574 & 0,70225 & 1,00000 \\
\hline eff_exp & 21 & 0,78669 & 0,07574 & 0,70225 & 1,00000 \\
\hline eff_hnx & 21 & 0,78669 & 0,07574 & 0,70225 & 1,00000 \\
\hline eff_expx & 21 & 0,78669 & 0,07574 & 0,70225 & 1,00000
\end{tabular}

Fonte: Elaborado pelos autores.

A variável eff_ols, conforme esperado, apresenta valor máximo maior que 1, pois a formulação MQO assume que todo o ruído estatístico está relacionado tanto com a omissão de variáveis relevantes do modelo quanto com os erros de medida e a ineficiência. A fronteira estocástica, por sua vez, permite que os valores estejam limitados acima, com valor máximo igual a um. Os escores eff_cols e eff_cmad apresentam uma correção no cálculo dos resíduos, de modo que há a garantia de que os valores de eficiência estarão dentro da fronteira de produção. Por isso que, no caso dos modelos COLS e CMAD, o máximo valor de eficiência é 1, conforme prescreve a teoria.

Nos modelos de fronteira estocástica, verifica-se a consistência da forma funcional proposta, uma vez que há coerência entre os escores de eficiência calculados por meio dos diversos mecanismos, reforçando o resultado do teste de distribuição dos resíduos comentado anteriormente.

Os resultados indicam haver uma diferença bastante relevante entre as estimativas paramétricas determinísticas OLS e COLS e as estimativas de fronteira estocástica half-normal e exponencial. A estimativa CMAD, por sua vez, se encontra mais próxima das de fronteira estocástica. Tal diferença está em conformidade com a literatura, uma vez que os métodos baseados em mínimos quadrados estão mais propensos a gerar resultados inconsistentes, ao contrário dos métodos baseados em máxima verossimilhança.

Os escores de eficiência, calculados na forma das equações (1) a (5), ou seja, excluindo outliers e calculando a média dos escores individuais obtidos por meio dos diferentes métodos, apresentam uma média relativamente alta de 0,84 , com valores máximo e mínimo de, respectivamente, 0,92 e 0,78 . A conclusão é que, em média, as concessionárias conseguem produzir $84 \%$ do output máximo permitido, sendo que a concessionária mais eficiente entrega $92 \%$.

\section{CONCLUSÃO}

Este trabalho teve como objetivo contribuir para o debate acerca dos mandamentos legais e contratuais impostos à ANTT no que tange ao incentivo à eficiência dos serviços prestados pelas concessionárias que atuam nas rodovias federais concedidas. 
A verificação da eficiência de monopólios regulados pelo Estado pode ser feita por meio da chamada análise de benchmarking, a qual consiste em avaliar de modo comparativo a eficiência das diversas firmas, baseando-se em parâmetros de entrada (input) e saída (output). O resultado da análise de eficiência, por sua vez, pode ser utilizado como ferramenta regulatória para incentivar as firmas reguladas a entregar melhores serviços aos usuários.

Para tanto, apresentou-se comparação entre diferentes métodos de mensuração de desempenho e suas variantes, presentes na literatura de regulação econômica. Foram cotejados variados métodos de benchmarking, com destaque para aqueles baseados na ideia de fronteira de eficiência, paramétricos (embasados em Mínimos Quadrados Ordinários, Stochastic Frontier Analysis e suas variações) e não paramétricos (Data Envelopment Analysis, puro, com correção de viés e em dois estágios).

Em que pese o pequeno número de unidades para análise (21 concessionárias), neste trabalho, buscou-se pautar a escolha das variáveis do modelo com apenas um output e dois inputs, de modo que este se adequasse à reduzida quantidade de dados. Foram, então, seguidas as regras disponíveis na literatura acerca da proporção entre inputs, outputs e o número de observações, o que garante a consistência dos resultados das análises, conforme exposto na seção referente aos dados do modelo.

Foi apresentado o arcabouço teórico que embasou a metodologia proposta, mostrando que as ferramentas escolhidas, apesar de já usadas por reguladores estrangeiros a alguns anos, representam o estado da arte na análise de benchmarking, restando clara a importância de se discutir a implementação sistemática de uma avaliação desse tipo nos contratos de concessão rodoviária no Brasil.

Com a estruturação fundamentada do modelo de benchmarking, com seus insumos e produtos, foram realizadas simulações por meio dos diversos métodos disponíveis para cálculo dos escores de eficiência de cada concessionária. A conclusão da análise por meio dos escores de eficiência é que, em média, as concessionárias conseguem produzir $84 \%$ do output máximo permitido, sendo que a concessionária mais eficiente entrega $92 \%$ deste.

Esse resultado é importante pois amplia a análise feita por artigos anteriores, considerando todos os contratos vigentes das três etapas de concessões rodoviárias federais e a aplicação de diversos modelos de benchmarking. Além disso, ao calcular os escores de eficiência, são fornecidos insumos para discussão de políticas públicas a serem adotadas no futuro e também para o comportamento do próprio agente regulador (ANTT), que pode atuar incentivando uma maior produtividade das concessionárias.

\section{REFERÊNCIAS}

AGRELL, P. J.; BOGETOFT, P. Benchmarking and regulation. Core Discussion Paper- Center for Operations Research and Econometrics, Université 
catholique de Louvain, CORE and Louvain School of Management, B-1348 Louvain-la-Neuve, Belgium, p. 23, 2013.

BANKER, R. D. et al. An Introduction to Data Envelopment Analysis With Some of Its Models and Their Uses, eds. J. Chan and J. Patton. Research in Governmental and Norz-Profit Accountirzg, v. 5, n. 125.163, 1989.

BOGETOFT, P.; OTTO, L. Benchmarking with Dea, Sfa, and R. [s.1.] Springer Science \& Business Media, 2010.

CLIMACO, J. C. N.; DE MELLO, J. C. C. S.; MEZA, L. A. A study of highways performance with a MOLP-DEA model and an interactive tricriteria linear programming package (TRIMAP). Brazilian Journal of Operations \& Production Management, v. 7, n. 1, p. 163-179, 2010.

COELLI, T. Estimators and hypothesis tests for a stochastic frontier function: A Monte Carlo analysis. Journal of productivity analysis, v. 6, n. 3, p. 247-268, 1995.

COELLI, T. J. et al. An introduction to efficiency and productivity analysis. [s.1.] Springer Science \& Business Media, 2005.

CORREA, C. A. V. Economic evaluation of current conditions of competition and efficiency of automotive and rail systems in Colombia. Energy Policy, v. 46, p. 78-87, 2012.

DIANA, T. Can we explain airport performance? A case study of selected New York airports using a stochastic frontier model. Journal of Air Transport Management, v. 16, n. 6, p. 310-314, 2010.

FARSI, M.; FILIPPINI, M. Regulation and measuring cost-efficiency with panel data models: Application to electricity distribution utilities. Review of Industrial Organization, v. 25, n. 1, p. 1-19, 2004.

FERREIRA, F. R. N. et al. Análise das concessões de rodovias federais utilizando DEA sob a perspectiva do usuário. In: XLV Simpósio Brasileiro de Pesquisa Operacional: a pesquisa operacional na busca de eficiência nos serviços públicos e/ou privados, Natal, 2013, v.16.

GOMES, A. P.; BAPTISTA, A. Análise envoltória de dados: conceitos e modelos básicos. Métodos quantitativos em economia. Viçosa: Editora UFV, p. 121-160, 2004.

GOMES, E. et al. Gestão de auto-estradas: análise de eficiência das autoestradas federais brasileiras com portagens. Revista de Gestão dos Países de Lingua Portuguesa, v. 11, n. 2-3, p. 55-62, 2012.

GUIMARÃES, F. C. V. Concessão de serviço público. [s.1.] Editora Saraiva, 2017. 
ISMAEL DE AZEVEDO, G. H. et al. Uso de análise envoltória de dados para mensurar eficiência temporal de rodovias federais concessionadas. Journal of Transport Literature, v. 6, n. 1, p. 37-56, 2012.

JAMASB, T.; POLLITT, M. Benchmarking and regulation: international electricity experience. Utilities policy, v. 9, n. 3, p. 107-130, 2000.

KUMBHAKAR, S. C.; WANG, H.; HORNCASTLE, A. P. A practitioner's guide to stochastic frontier analysis using Stata. [s.1.] Cambridge University Press, 2015.

LAFFONT, J.-J.; TIROLE, J. A theory of incentives in procurement and regulation. [s.l.] MIT press, 1993.

MASSIANI, J.; RAGAZZI, G. Costs and efficiency of highway concessionaires: a survey of Italian operators. European Transport n. 38, pp. 85-106, 2008.

MEDEIROS, F. DA S. Eficiência em concessões de infraestrutura: Benchmarking, Price-cap e o fator " $x$ ". 2014. Dissertação de Mestrado (Programa de PósGraduação em Engenharia de Produção). Porto Alegre, RS: Universidade Federal do Rio Grande do Sul.

NETO, A. S. Estatística e Introdução à Econometria. [s.1.] Editora Saraiva, 2017.

PICOT, A. The Economics of Infrastructure Provisioning: The Changing Role of the State. [s.1.] MIT press, 2015.

POSNER, R. A. Teorias da regulação econômica. Regulação econômica e democracia: o debate norte-americano. São Paulo: Editora, v. 34, p. 49-80, 2004.

POSSAMAI, R. P. Avaliação de eficiência técnica em concessionárias de rodovias utilizando Análise Envoltória de Dados. 2006. Dissertação de Mestrado (Programa de Pós-Graduação em Engenharia de Produção). Porto Alegre, RS: Universidade Federal do Rio Grande do Sul.

PROFETA, G. A. Regulação e eficiência dos modelos de concessões de rodovias no Brasil. 2014. Tese de Doutorado (Programa de Pós-Graduação em Economia Aplicada) Viçosa, MG: Universidade Federal de Viçosa.

RAMALHO, E. A.; RAMALHO, J. J.; HENRIQUES, P. D. Fractional regression models for second stage DEA efficiency analyses. Journal of Productivity Analysis, v. 34, n. 3, p. 239-255, 2010.

SALGADO, S. D. R.; WILBERT, M. D.; ROSANO, C. Concessões Rodoviárias Federais: Análise da Eficiência da Primeira e Segunda Etapa dos Contratos de Concessão. . In: XXIII SIMPEP - GESTÃO DE OPERAÇÕES EM SERVIÇOS E SEUS IMPACTOS SOCIAIS. Bauru, SP: 2016. 
SCOTTI, D. et al. The impact of airport competition on technical efficiency: A stochastic frontier analysis applied to Italian airport. Journal of Air Transport Management, v. 22, p. 9-15, 2012.

SIMAR, L.; WILSON, P. W. Estimation and inference in two-stage, semiparametric models of production processes. Journal of econometrics, v. 136, n. 1, p. 31-64, 2007.

SMITH, A. S. The application of stochastic frontier panel models in economic regulation: Experience from the European rail sector. Transportation Research Part E: Logistics and Transportation Review, v. 48, n. 2, p. 503-515, 2012.

TIROLE, J. Economics for the common good. [s.1.] Princeton University Press, 2017.

TIRYAKI, G. F.; ANDRADE, C. S. M. Econometria na prática. 1a. ed. Rio de Janeiro, RJ, Brasil: Alta Books, 2017.

WANG, L.-C.; TSAI, H.-Y. Evaluation of Highway Maintenance Performance Using Data Envelopment Analysis (DEA) in Taiwan. Journal of Marine Science and Technology, v. 17, n. 2, p. 145-155, 2009.

WELDE, M.; ODECK, J. The efficiency of Norwegian road toll companies. Utilities Policy, v. 19, n. 3, p. 162-171, 2011. 